\title{
Calibration and Validation of a Skeletal Multibody Model for Leg-Orthosis Contact Force Estimation
}

\author{
Francisco Mouzo ${ }^{1}$, Urbano Lugris ${ }^{1}$, Javier Cuadrado ${ }^{1(\bowtie)}$, \\ Josep M. Font-Llagunes ${ }^{2}$, and Francisco J. Alonso ${ }^{3}$ \\ ${ }^{1}$ Laboratory of Mechanical Engineering, University of La Coruña, Ferrol, Spain \\ javier.cuadrado@udc.es \\ 2 Department of Mechanical Engineering and the Biomedical Engineering \\ Research Centre, Technical University of Catalonia, Barcelona, Spain \\ ${ }^{3}$ Department of Mechanical, Energetics and Materials Engineering, \\ University of Extremadura, Badajoz, Spain
}

\begin{abstract}
Estimation of contact forces between lower limb and orthosis during gait is useful to prevent skin issues in subjects wearing this type of assistive devices. While inverse-dynamics based gait analysis of multibody models is difficult to apply due to the limited accuracy of motion capture systems, a forward-dynamics based analysis in which leg and orthosis are considered as independent entities is shown to provide acceptable results. Contact model parameters are calibrated through comparison of measured and calculated bending torque at the orthosis location where a load cell is installed, and the attained correlation allows to validate the model.
\end{abstract}

\section{Introduction}

The authors developed an active knee-ankle foot orthosis (KAFO) as an assistive device for the gait of spinal cord injured (SCI) subjects [1]. The prototype (Fig. 1a) features a brushless DC motor at knee level to provide knee motion during the swing phase and an inertial sensor at shank level to detect motion intention and trigger the swing cycle.

For bilateral patients, the control algorithm that launches the orthosis swing cycle is based on the orientation of both shanks obtained after processing data coming from the two inertial sensors. To have the maximum time to complete the cycle before the foot touches down again, the swing cycle must be launched as soon as motion intention is detected, but false positives cannot be allowed due to fall risk.

For unilateral patients (Fig. 1b), one single inertial sensor is available, which means less information to detect motion intention. Therefore, a load cell is included in the

This work was funded by the Spanish MINECO under project DPI2015-65959-C3-1-R, cofinanced by the EU through the EFRD program, and by the Galician Government under grant ED431B2016/031. 

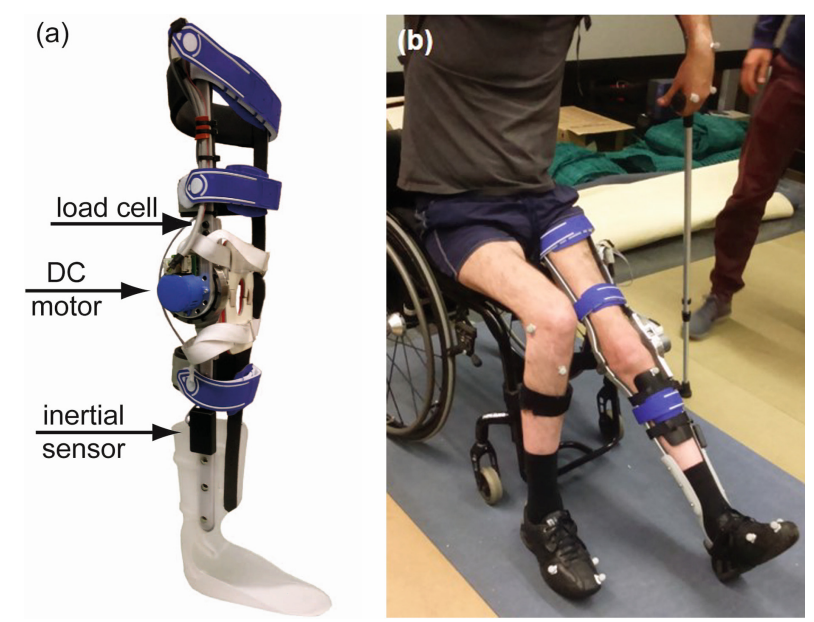

Fig. 1. Prototype of active knee-ankle-foot orthosis: (a) actuator and sensors; (b) unilateral patient wearing the orthosis.

orthosis structure to alleviate this problem (while avoiding the many issues raised by pressure sensors): it is formed by two strain gauges located in the front and rear faces of the external orthosis upright at thigh level, just above the DC motor. The load cell detects the increment in load suffered by the orthosis during stance, as the knee bends under the subject's weight and the leg presses against the orthosis. This serves to reliably detect stance and, hence, provides the necessary information to guarantee a safe orthosis operation.

In this work, the load cell is used to calibrate and validate a computational multibody model of a subject wearing the orthosis which includes a leg-orthosis contact model.

\section{Materials and Methods}

An experiment was conducted with a 49-year-old adult spinal-cord-injured male, mass $82 \mathrm{~kg}$ and height $1.90 \mathrm{~m}$, requiring, in order to walk, a KAFO on his left leg and an ankle-foot orthosis on his right leg, along with a pair of crutches (Fig. 1b). In the experiment, he was wearing our active KAFO in his left leg, and walked over two embedded force plates (AMTI, AccuGait sampling at $100 \mathrm{~Hz}$ ) with the help of two instrumented crutches that measured the ground contact forces at their tips. Motion was captured by 12 optical infrared cameras (Natural Point, OptiTrack FLEX:V100 also sampling at $100 \mathrm{~Hz}$ ) that computed the position of 43 optical markers. The study was approved by the institutional ethical committee and the subject gave his informed consent.

A 59 degree-of-freedom multibody model of the subject wearing orthoses and crutches was developed as in [2] but, this time, links of active orthosis and left leg were modeled as independent entities (Fig. 2a). Hence, relative motion and contact forces 
between leg and orthosis could be estimated when running a forward dynamic simulation of the model that tracked the subject's captured motion through a CTC control scheme [3]. Figure 2b illustrates the modeling of leg and orthosis in the multibody model, along with the contact spring-damper elements at hip and knee levels.
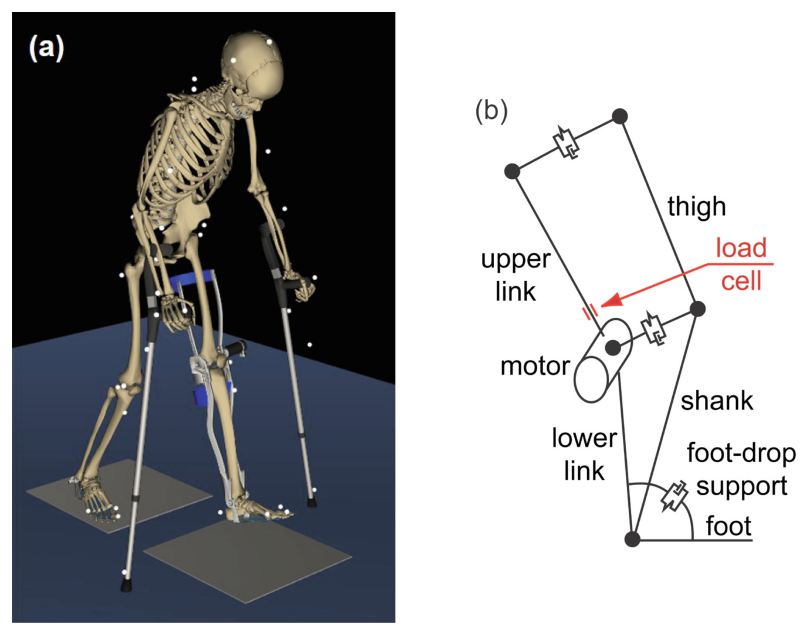

Fig. 2. Model of unilateral patient with active orthosis: (a) graphical output; (b) modeling of leg and orthosis as independent entities.

In a previous work [4], the variations of leg-orthosis contact forces and misalignments for bilateral subjects were studied as functions of the stiffness/damping parameters of the contact elements. Figure 3 shows the interaction forces at right hip level for three different values of the parameters for the case of a bilateral SCI female. The blue and pink areas correspond to the swing phase of the right and left leg,

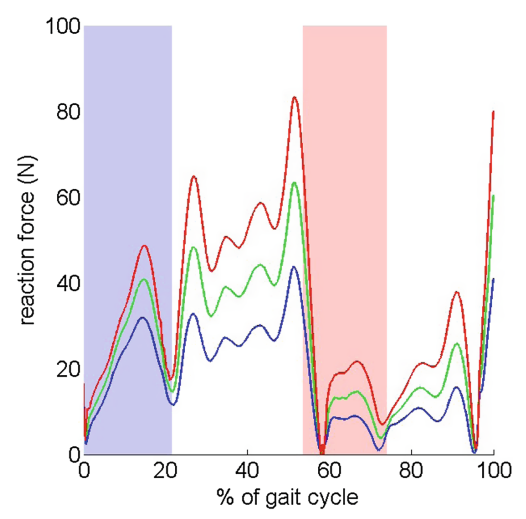

Fig. 3. Contact forces between leg and orthosis at hip connection for three different values of the parameters of the contact elements. 
respectively. The curves show a similar shape for the three values of the parameters, just differing in a vertical scaling.

In this work, to calibrate the stiffness/damping parameters the load cell was used. It measures the strain due to axial stresses caused by the bending effects of the forces acting on the KAFO above the location of the gauges, i.e. leg-orthosis contact force at the thigh strap and weight plus inertia forces of the orthosis thigh body (Fig. 2b). The load cell was calibrated with known loads, so as to make it a transducer of the bending torque. Then, the history of such magnitude measured in the experiment was compared with that calculated for different values of the contact model parameters, looking for a good correlation, so as to calibrate the stiffness/damping of the contact elements.

\section{Results}

Figure 4 shows the history of the bending torque at the load cell location obtained by measurement and calculation using the best set of the contact parameters selected in several manual iterations. The RMS error is $2.83 \mathrm{Nm}$.

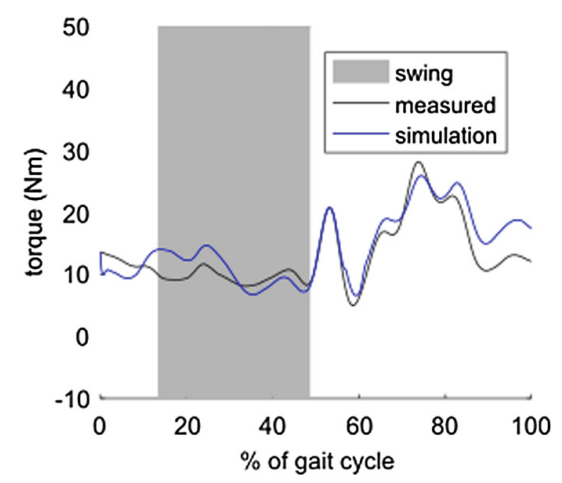

Fig. 4. Comparison of the torque measured by the load cell and calculated from the model.

\section{Discussion}

Results shown in the previous section confirm the validity of the proposed model and analysis method to estimate the contact forces between leg and orthosis during gait. It must be pointed out that, while contact forces could be hardly obtained through an inverse-dynamics based approach, as it is not easy to discriminate the motions of leg and orthosis with the accuracy provided by current motion capture systems, application of a forward-dynamics based approach, in which only the motion of leg or orthosis is measured and the counterpart is left to its own dynamics, provides acceptable results. 


\section{Conclusions}

In this work, it has been demonstrated that, for subjects wearing knee-ankle-foot orthoses and crutches, the contact forces between leg and orthosis during gait can be reasonably estimated through a forward-dynamics based analysis of a full multibody model in which legs and orthoses are considered as independent entities.

\section{References}

1. Font-Llagunes, J.M., Clos, D., Lugris, U., Alonso, F.J., Cuadrado, J.: Design and experimental evaluation of a low-cost robotic orthosis for gait assistance in subjects with spinal cord injury. In: Gonzalez Vargas, J., et al. (eds.) Wearable Robotics: Challenges and Trends, pp. 281-286. Springer (2016)

2. Lugris, U., Carlin, J., Luaces, A., Cuadrado, J.: Gait analysis system for spinal cord injured subjects assisted by active orthoses and crutches. J. Multi-body Dyn. 227, 363-374 (2013)

3. Mouzo, F., Lugris, U., Pamies-Vila, R., Cuadrado, J.: Skeletal-level control-based forward dynamic analysis of acquired healthy and assisted gait motion. Multibody Syst. Dyn. 44(1), 1-29 (2018)

4. Mouzo, F., Lugris, U., Cuadrado, J., Font-Llagunes, J.M., Alonso, F.J.: Evaluation of motion/force transmission between passive/active orthosis and subject through forward dynamic analysis. In: Ibañez, J., et al. (eds.) Converging Clinical and Engineering Research on Neurorehabilitation II, pp. 815-819. Springer (2016) 